\title{
Increased Risk for Neurodevelopmental Disorders in Children With Orofacial Clefts
}

\author{
Karin K. Tillman, MD, Malin Hakelius, MD, PhD, Jonas Höijer, MSc, Mia Ramklint, MD, PhD, \\ Lisa Ekselius, MD, PhD, Daniel Nowinski, MD, PhD, Fotios C. Papadopoulos, MD, PhD
}

Objective: Children with orofacial clefts (OFC) may have an increased risk of poor mental health. This study aimed to investigate the risk of psychiatric diagnoses in individuals with OFC, stratified by cleft type.

Method: A nationwide register-based cohort of all individuals born with nonsyndromic OFC in Sweden between 1973 and 2012 ( $\mathrm{n}=7,842$ ) was compared to a matched cohort $(\mathrm{n}=78,409)$ as well as to their unaffected siblings $(\mathrm{n}=9,637)$. The risk of psychiatric diagnoses, suicide attempts, and suicides was examined by crude and adjusted Cox regression models. Effect modification by sex was investigated with interaction terms in the models.

Results: Children with cleft lip (CL) had a significantly higher risk of any psychiatric disorder, intellectual disability, and language disorders; children with cleft lip and palate (CLP) had, in addition, an increased risk of autism spectrum disorder (ASD). Children with cleft palate only (CPO) had risk increases for the same diagnoses as children with CL and CLP, but with higher hazard ratios, and also for psychotic disorders, attention-deficit/ hyperactivity disorder (ADHD), and other behavioral or emotional disorders in childhood. Sex stratification indicated higher risk increases among females in CL and CLP but not in CPO. Siblings without OFC were less likely to be diagnosed with any psychiatric disorder, intellectual disability, language disorder, ASD, or ADHD compared to their siblings with OFC.

Conclusion: Children with nonsyndromic clefts had a significantly higher risk of neurodevelopmental disorders. This risk is unlikely to be explained by familial influences such as inherited genetic or shared environmental factors.

Key words: epidemiology, nonsyndromic clefts, psychiatric comorbidity, neurodevelopmental disorders

J Am Acad Child Adolesc Psychiatry 2018;57(11):876-883.

CME (2)

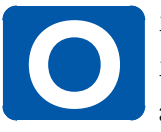
rofacial clefts (OFC) are one of the most common congenital malformations. ${ }^{1}$ OFC arise in about 1 per 500 live-born infants in Sweden. ${ }^{2}$ The OFC are, on an anatomical basis, grossly divided into three different subtypes of clefts; cleft lip (CL), cleft lip and palate (CLP), and cleft palate only (CPO), in which $\mathrm{CPO}$ is pathophysiologically regarded as a separate condition in relation to CLP and CL. Approximately $30 \%$ of $\mathrm{OFC}$ are associated with a known genetic syndrome (syndromic OFC), but the remaining $70 \%$ of clefts occur without a known syndrome identified (nonsyndromic OFC). ${ }^{3,4}$ More than 300 cleft-related syndromes have been described, and CPO is more likely to be syndromic than CL and CLP. OFC occur at gestational week 5 to 8 , when the neural crest cells differentiate from the neural tube and migrate to the facial region. Inheritance may be chromosomal, sporadic, or related to environmental teratogens., Known maternal risk factors include smoking, ${ }^{7,8}$ alcohol consumption, ${ }^{8}$ diabetes, nutritional factors (eg, vitamin A, folic acid insufficiency), and anticonvulsant medication. ${ }^{9}$
Depending on the cleft type, different concerns for the child may arise. Children with OFC need multidisciplinary care from birth to adulthood, including staged surgical treatment as well as monitoring of the various cleft-associated symptoms and features such as difficulties with feeding, hearing, dento-facial growth, speech, and appearance. Multidisciplinary care for OFC with long-term follow-up until the age of 19 years was established in Sweden during in the 1960s and today is conducted through 6 regional cleft lip and palate centers. Surgical protocols have gradually evolved over time, and with some variations in surgical techniques among centers. The longitudinal assessments of facial growth and speech today are, to large extent, harmonized nationally through the network Swedecleft. All Swedish children are monitored at the primary care level for general growth, hearing, vision, and development according to national guidelines.

Another area of concern is cognitive development and language skills. Cognitive dysfunction in children with OFC has been documented over time. ${ }^{10}$ In addition, nonsyndromic clefts have been found to be associated with poor academic 
achievement, a lower verbal IQ, and deficits in rapid verbal labeling, verbal fluency, and short-term memory. ${ }^{11}$ Among children with nonsyndromic OFC, the prevalence of learning disabilities, particularly specific reading disorders, has been estimated to be between $30 \%$ and $46 \%{ }^{12,13}$ Less attention has been placed on executive functioning skills (attention, organization, monitoring, planning, and initiation) or psychiatric disorders. Moreover, most previous studies have been based on small samples in a clinical setting, with only a few of them distinguishing among the cleft subtypes. Earlier research has found different patterns of deficits for patients with CL, CLP, and $\mathrm{CPO}$, with more severe disabilities reported in the $\mathrm{CPO}$ group. Children with CLP have shown more deficits in verbal expressive skills, whereas children with $\mathrm{CPO}$ have demonstrated poorer associative and expressive language skills. ${ }^{14}$ Recent work suggests that frontal and prefrontal function may be impaired in many children with clefts, and further examination of executive functions during follow-up is recommended. ${ }^{15}$

Danish adults born with OFC have been reported to have an increased risk for inpatient treatment for psychiatric disorders, ${ }^{16}$ as well as increased all-cause mortality and suicide. ${ }^{17}$ A recent extension of this Danish study, in which outpatient visits and emergency room visits were also included and a differentiation among the various types of clefts was made, pointed out a significantly increased risk for psychiatric disorders among individuals with nonsyndromic OFC compared to controls. ${ }^{18}$ Recently, a large Swedish population-based study showed that CL and CPO increase the risk for psychotropic drug use in adolescence; however, there was no distinction made among the different groups of psychotropic drugs or psychiatric indications. ${ }^{19}$ The majority of previous studies are small, underpowered, and clinically based, and focus on psychological symptoms rather than psychiatric disorders as a medical diagnosis.

The aim of this study was to test the hypothesis that patients with nonsyndromic OFC have an increased risk of a psychiatric diagnosis later in life. As a secondary aim, we wanted to explore whether such an increased risk could be explained by familial influences such as inherited genetic or shared environmental factors, by studying psychiatric outcomes among the unaffected full siblings of the children with OFC.

\section{METHOD}

\section{Data Sources}

Data were obtained from the National Board of Health and Welfare in Sweden and Statistics Sweden. All registers use the 10-digit National Registration Number (NRN), a unique personal identifier assigned to all Swedish residents, which allows linkage studies. In the data that we received, identification numbers were replaced with arbitrary numbers, thereby securing anonymity. The following registers were used: the Swedish Medical Birth Register (MBR), the National Patient Register (NPR), the Swedish Cause-of-Death register, the Register of Total Population (RTP), the Multi-Generation Register (MGR), the Migration Register, the Census of the population and housing, and the longitudinal integration database for health insurance and labor market studies (LISA).

The MBR has information on more than $99 \%$ of all births in Sweden since 1973, concerning pregnancy, delivery, and the neonatal period. Information is collected from medical records from the prenatal, delivery, and neonatal care, and includes data such as maternal diagnoses before and during pregnancy, tobacco use, alcohol use, medical drug use, mode of delivery, analgesia and anaesthesia, birth weight, body length, head circumference, duration of pregnancy, Apgar score, and infant diagnoses. ${ }^{20}$

The NPR has nearly complete nationwide coverage for discharge diagnoses in both somatic and psychiatric settings in Sweden based on the International Classification of Diseases. It has full coverage of all inpatient care in Sweden since 1987. Each record includes admission and discharge dates, the main discharge diagnosis, and secondary diagnoses. Outpatient specialist visits, including day surgery and psychiatric care from both private and public caregivers, have been included since 2001, and the coverage has increased from approximately $75 \%$ initially to $87 \%$ in 2011 . $^{21}$

The Swedish Cause-of-Death Register includes all individuals who died either in Sweden or abroad since 1952 and who were registered in Sweden by the time of death. The data are based on death certificates that provide information on date as well as underlying main and secondary causes of death using the International Classification of Diseases (ICD). ${ }^{22}$

The Register of Total Population includes information about the country of birth.

Data available from the Swedish Multi-Generation Register allow for linkage of individuals born in 1932 and later to parents, siblings, and offspring. Only pregnancies leading to live births can be identified in the Swedish MultiGeneration Register. ${ }^{23}$

The Uppsala Ethics Committee approved the study (Reg. No. 2012/363).

\section{Participants}

We identified, through MBR and NPR, 7,900 children born in Sweden between January 1, 1973, and December 31,2012 , who received the diagnosis of OFC at birth or prior to 5 years of age.

Two comparison groups were used: (1) 10 individuals without diagnosis for OFC malformation from the general population, matched according to the month and year of 
birth, sex, and county of birth; and (2) all unaffected siblings of the OFC patients with less than 20 years' age difference, identified in the Multi Generation Register, enabling us to control for familial influences such as inherited genetic or shared environmental factors.

We excluded 58 individuals without any identified matched individuals with whom they could be compared.

Comorbid diagnoses of any congenital malformations, deformations, and chromosomal abnormalities (ICD 8 and -9 codes 740-759 and ICD-10 codes Q00-Q99, except for the facial cleft codes [749 in ICD-8 and -9 and Q35-Q37 in ICD-10]) were identified as syndromic indicators in both cases and comparison groups, to adjust the analyses for possible nonidentified syndromic OFC.

All subjects were observed from their date of birth until outcome, emigration, death, or end of the study on December 31, 2012, whichever occurred first.

\section{Exposure}

The exposure was a diagnosis of CL, CLP, or CPO as indicated in the MBR or NPR by their ICD-8, ICD-9, or ICD-10 diagnoses. The ICD codes for CL were 749.10-749.13 (ICD-8), 749B (ICD-9), and Q36 (ICD10); for CLP the codes were 749.20-749.24 (ICD-8), 749C (ICD-9), and Q37 (ICD-10); and for CPO the codes were 749.00 (ICD-8), 749A (ICD-9), and Q35 (ICD-10).

\section{Outcome Measures}

Information on psychiatric diagnoses and suicide attempts was extracted from the NPR, and suicides were extracted from the Swedish Cause-of-Death Register. We studied the following psychiatric diagnoses (see Table S1, available online, for the respective ICD codes): any psychiatric disorder, intellectual disability (including mental retardation in ICD-8), speech and language disorders, psychotic disorders, bipolar disorder, depression, autism spectrum disorders (ASD), attention-deficit/hyperactivity disorder (ADHD), as well as other behavioral/emotional disorders with onset in childhood; neurotic, stress-related, or somatoform disorder; eating disorders; alcohol and substance use disorders; personality disorder; suicide attempt; and suicide.

When analyzing the mean age at first diagnosis for each of the above psychiatric disorders, we noticed a clear discrepancy for the age at first diagnosis with an eating disorder, namely a mean age of 7.8 years among children with OFC and 14.5 years among the children without OFC. As the ICD-8 and ICD-9 codes for eating disorders were not specific, we considered those events as probable feeding difficulties early in life misclassified as eating disorders. Thereafter we restricted the diagnosis of eating disorder to those who received such a diagnosis at the age of 10 years or older.

\section{Covariates}

We controlled the multivariate analyses for confounders and mediators such as perinatal complications and somatic indicators, year and season of birth, sex, congenital malformations or known genetic syndromes, parental psychiatric morbidity, and sociodemographic factors among parents.

Perinatal variables were collected from the MBR. Gestational age at birth was dichotomized into term birth ( $\geq 37$ gestational weeks) or preterm birth $(<37$ gestational weeks). Small for gestational age was defined as less than -2 SD. Birth weight was defined as low if $<2,500 \mathrm{~g}$. Low Apgar score was defined as $<7$ at 5 minutes after birth. A binary variable was created for gestational complications (preterm, small for gestational age [SGA], low Apgar, and low birth weight) and was used in the models. Season of birth was categorized as winter (December to February), spring (March to May), summer (June to August), and autumn (September to November).

Sociodemographic variables and parental mental health variables were accessed through linkage via the MGR to the biological parents. Ages of parents at the time of birth were identified, and we used the mean age of the parents or the age of one parent if the other's was missing for the multivariate analyses.

Data on maternal country of birth from the MBR were aggregated across regions: Sweden, other Nordic countries, and other countries. Information on the educational level of parents was retrieved from the Education Register, the LISA database, and the Census of the population and housing from the year 1985. Parental education was entered into the model as a categorical variable using 5 categories according to the Swedish Education Terminology: 0 to 9 years, 10 to 11 years, 12 to 14 years, and $>14$ years (university). The highest level of education obtained by either of the parents was used in the analysis.

Parental psychiatric morbidity was defined as having at least one psychiatric diagnosis (codes 290-315 in ICD-8, 290-319 in ICD-9, and F00-F98 in ICD-10) in the NPR; a suicide attempt (codes E950-E959 in ICD-8 and ICD-9, and X60-X84 in ICD-10) in the NPR; or a death by suicide in the Cause-of-Death Register. This ordinal variable would take the value of 0 if none of the parents had psychiatric morbidity, 1 if only one parent had psychiatric morbidity and 2 of both parents had psychiatric morbidity. The variable was treated as time varying in the analyses.

\section{Statistical Analysis}

The data were analyzed using the statistical software Stata v.13. ${ }^{24}$ Crude and adjusted Cox proportional hazard regression models were used with age as the underlying time scale to investigate hazard ratios and $95 \%$ CIs for the 
outcomes. Because the controls and siblings could not be considered as independent of the corresponding case, cluster robust variance-covariance estimation was used. ${ }^{25} \mathrm{We}$ compared the individuals with OFC with both the matched comparison cohort and the sibling cohort. Additional analyses were stratified by cleft type. For the continuous covariates concerning parental age and year of birth, we used restricted cubic spline to avoid forcing the relationship to the outcomes to be linear.

To examine a possible moderating effect of sex, we estimated separate Cox models in which an interaction term of sex and exposure (OFC, CL, CLP, CPO) was included. From these models, the $p$ value of the interaction term was calculated. However, some of these models were not possible to estimate because of too rare outcomes in one of the two sexes.

\section{RESULTS}

Descriptive characteristics of the study population for the studied cohorts are provided in Table S2, available online. A total of 7,842 children with OFC and 78,409 matched children without OFC were observed for 1,654,328 person-years. Among the children with OFC, 2,526 (32\%) were diagnosed with CL; 3,048 (39\%) were diagnosed with CLP; and 3,436 (43\%) were diagnosed with CPO. Almost 20\% of the children with OFC (CL, 15.4\%; CLP, $19.1 \%$; and CPO, 23.2\%) received at least one diagnosis of a psychiatric disorder, compared to $11 \%$ of the children without OFC.

More specifically, children with OFC were more likely to receive a diagnosis of any psychiatric disorder $(\mathrm{aHR}=1.63,95 \% \mathrm{CI}=1.53-1.73)$, intellectual disability $(\mathrm{aHR}=4.19,95 \% \mathrm{CI}=3.63-4.84)$, language disorder $(\mathrm{aHR}=4.89,95 \% \mathrm{CI}=4.08-5.87), \mathrm{ASD}(\mathrm{aHR}=1.96$, $95 \% \mathrm{CI}=1.65-2.33)$, psychotic disorder $(\mathrm{aHR}=1.62$, $95 \% \mathrm{CI}=1.15-2.29)$, $\mathrm{ADHD}(\mathrm{aHR}=1.25,95 \% \mathrm{CI}=$ 1.08-1.46), other behavioral and emotional disorders with onset in childhood $(\mathrm{aHR}=1.43,95 \% \mathrm{CI}=1.23-1.65)$, and personality disorders $(\mathrm{aHR}=1.35,95 \% \mathrm{CI}=$ 1.00-1.82) (Figure 1, Tables S3-S6, available online).

Children with cleft lip (CL) had a significantly higher risk of any psychiatric disorder, intellectual disability, and language disorders. Children with cleft lip and palate (CLP) also had an increased risk of ASD. Children with cleft palate only (CPO) presented with higher hazard ratios, and additionally for psychotic disorders, $\mathrm{ADHD}$, and other behavioral or emotional disorders in childhood (Figure 1A-D, Tables S3-S6). Interestingly, children born with CL presented with a risk decrease for depression $(\mathrm{aHR}=0.73,95 \% \mathrm{CI}=0.54-0.98)$.
No significant risk increase was found among any of the stratified cleft types for suicide attempts, death by suicide, affective disorders, neurotic or anxiety disorders, eating disorders, alcohol or psychoactive substance abuse, and a modest but significant risk increase for personality disorders was seen only in the OFC analysis.

In the sex-specific analyses, we found, within the OFC cohort, risk increases that were significantly higher among females than among males for any psychiatric disorder (interaction $p=0.007$ ), intellectual disability (interaction $p=0.017$ ), language disorders (interaction $p=0.023$ ), ASD (interaction $p=0.015$ ), and other behavioral and emotional disorders with onset in childhood (interaction $p=0.005)$, whereas the sex difference was borderline significant for psychotic disorders (interaction $p=0.089$ ) and ADHD (interaction $p=0.089$ ) and not significant for personality disorder (interaction $p=0.5$ ) Our analyses also detected a borderline significant sex difference among individuals born with CL and the risk of depression, whereby mostly the males presented with a risk decrease for depression $(\mathrm{aHR}=0.54,95 \% \mathrm{CI}=0.33-0.87$, interaction $p=0.081$ ) (Figure 1, Tables S3-S6, available online). These sex differences with generally higher risk increases for females persisted in some of the analyses after stratification into the different cleft types, and most evidently among children born with CLP.

A total of 9,637 full siblings to our individuals with OFC were studied in the sibling cohort. The unaffected full siblings without OFC were less likely to be diagnosed with any psychiatric disorder, intellectual disability, language disorder, ASD, and ADHD, whereas no significant different risk pattern was observed for psychotic disorders, other behavioral disorders in childhood, and personality disorders (Figure 1, Tables S7-S10, available online).

\section{DISCUSSION}

In this large Swedish nationwide register cohort study, we found a significant risk increase for being diagnosed with a psychiatric disorder, and specifically with intellectual disability, language disorder, ASD, ADHD, psychotic disorder, other behavioral and emotional disorders with onset in childhood, and personality disorders, among children born with an OFC compared with children without an OFC. Our sibling analyses confirmed decreased risks for any psychiatric disorder, intellectual disability, language disorders, ASD, and ADHD among siblings without OFC, suggesting that the associations with these diagnoses are unlikely to be explained by familial influences. There was no significant increase in suicide attempts or suicides, and no increased risk of anxiety disorders, depression, bipolar 
FIGURE 1 Adjusted Cox-Derived Hazard Ratios (aHRs) With 95\% Cls for Psychiatric Diagnoses, Suicide Attempts, and Suicide Among Children With Orofacial Clefts (OFC) Compared to Children Without Clefts
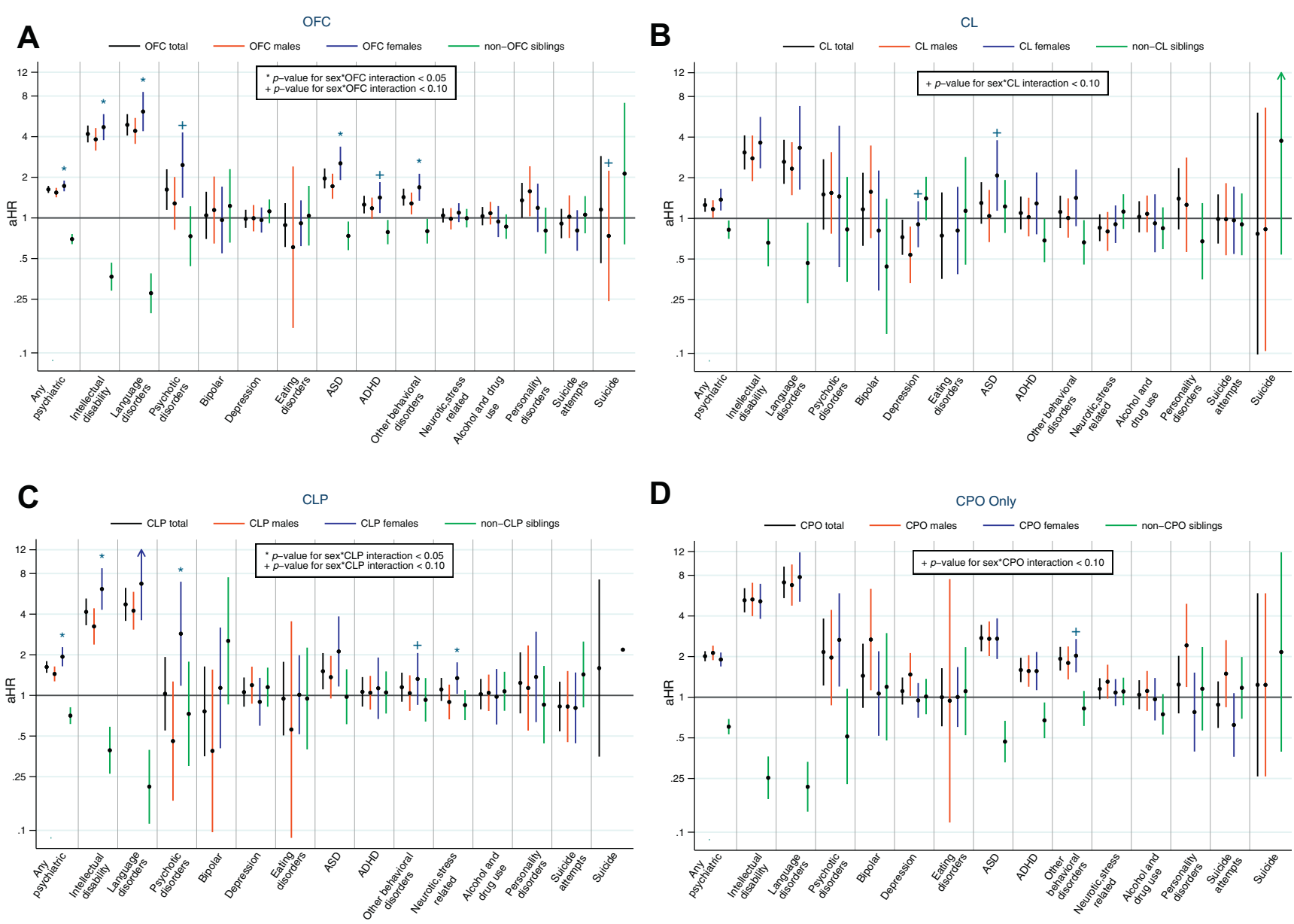

Note: aHRs are presented separately for males and females as well as for siblings without clefts compared to their full siblings with clefts. (A) Children with OFC. (B) Children with cleft lip (CL). (C) Children with cleft lip and palate (CLP). (D) Children with cleft palate only (CPO). ADHD = attention-deficit/hyperactivity disorder; $A S D=$ autism spectrum disorder. Please note color figures are available online.

disorder, eating disorders, or alcohol or substance use disorder.

When investigating the different subtypes of OFC, the risk increases differed. The overall risk increase for psychiatric morbidity was highest among individuals with CPO and lowest among those with CL. Those findings remained significant when adjusted for sociodemographic, perinatal, and somatic covariates. Our findings are in line with the results from a previous large population-based cohort study from Denmark in which a higher risk of psychiatric disorders emerged among individuals with nonsyndromic OFC, and especially for CPO, when stratifying for different cleft types. The Danish study also revealed a higher mortality in suicide, a finding that was not seen in our study. ${ }^{18}$ Interestingly, our study revealed substantially higher risk increases for all neuropsychiatric diagnoses compared with the Danish study. A plausible explanation could be a higher detection rate of neurodevelopmental disorders in the OFC population in Sweden and possibly more support services following the diagnosis. This would also be relevant when interpreting the higher risk of suicide and suicide attempts seen in Denmark, but not in Sweden, among individuals with OFC.

The highest risk increases among children with nonsyndromic OFC were seen for the diagnoses intellectual disability and language disorders, followed by ASD and psychosis. An increased risk for an ADHD diagnosis was seen among children with $\mathrm{OFC}$ and $\mathrm{CPO}$, whereas an increased risk for an ASD diagnosis was evident in all cleft types except for CL, in which the increased risk was statistically significant only among females. There was an interesting, notable discrepancy in the sizes of the risk increases for the different psychiatric diagnoses. The risk increase for psychiatric diagnoses other than intellectual disability and language disorders among OFC, and within 
each subtype (CL, CLP, or CPO) were approximately half of that risk, suggesting a stronger neurodevelopmental association for intellectual disability and language disorders.

There is emerging evidence that bipolar disorder and schizophrenia have a common genetic pathway, and we expected a risk increase not only for psychotic disorders but also for bipolar disorder. ${ }^{26,27}$ Our results with an increased risk only for psychotic disorders may support the notion of different trajectories for schizophrenia and bipolar disorder leading to different phenotypes. Another plausible explanation could be the fact that bipolar disorder was diagnosed less frequently in previous years and other diagnoses were used instead, and this could have obscured a risk increase in our study. Moreover, the mean age at a diagnosis of bipolar disorder in our study was approximately 25 years among individuals with OFC and their matched nonaffected individuals, whereas the respective age for a diagnosis of psychotic disorder was 23 years. Thus, it can be assumed that the age of our cohort could have been a restricting factor in capturing more diagnoses of bipolar disorder. A larger study population or a longer follow-up period could possibly detect and reveal an association between OFC and bipolar disorder.

The cognitive deficits and poorer mental health among children born with OFC have earlier been attributed to secondary factors, including low self-esteem, depressed mood, and hearing and speech deficits. ${ }^{28-30}$ Psychological factors in the child's environment are crucial for the child's mental development. However, it is possible that these deficits are, instead, the result of an abnormal brain development. Our finding of no increased risk for mood disorders or anxiety-related disorders, which are consistent with the recent results from Denmark, support the hypothesis of neurodevelopmental differences as a major common pathophysiologic mechanism for the association between OFC and psychiatric disorders. ${ }^{18}$

It has been suggested that facial malformations may be associated with aberrant brain development. ${ }^{31}$ Interestingly, associations between nonsyndromic OFC and structural brain anomalies have been reported in children and adolescents with clefts. $^{32,33}$ These midline brain anomalies have been associated with intellectual disability, developmental delay, and schizophrenia in previous studies. ${ }^{34,35}$ The relatively high incidence of central nervous system abnormalities among individuals with nonsyndromic OFC, being approximately 13 times higher compared to that in the general population, ${ }^{36}$ provides additional support for the hypothesis that cognitive and language deficits as well as other psychiatric disorders may be the result of underlying neurodevelopmental abnormalities. The craniofacial skeleton and the brain are derived from the same ectodermal tissues, and their development is closely linked during early morphogenesis. ${ }^{37,38}$
To further elucidate the possible mechanisms behind the association between OFC and neurodevelopmental disorders, we studied all unaffected full siblings. Children without OFC were less likely, compared to their siblings with any OFC or $\mathrm{CPO}$, to be diagnosed with any psychiatric disorder, intellectual disability, language disorder, ASD, and ADHD, whereas no significant different risk pattern was observed for psychotic disorders, other behavioral disorders in childhood, and personality disorders. Siblings without OFC, compared to their siblings with CL or CLP, had a decreased risk only for any psychiatric disorder, intellectual disability, and language disorders. The results from the siblings' analyses suggest that the strong associations with intellectual disability and language disorders and the modest associations with ASD and ADHD are unlikely to be explained by familial influences such as inherited genetic or shared environmental factors, supporting the theory that these neuropsychiatric diagnoses and OFC might be the result of the same abnormal neurodevelopment.

Interestingly, we also found a risk decrease for depression among children with CL, and the risk decrease was seen mostly among males with CL. This finding, which may be a chance finding, is difficult to interpret in terms of a biological or psychological resilience for the development of major depression in children with CL, especially as males with $\mathrm{CPO}$ showed an increased risk for depression. A rather more plausible explanation could be that males with CL are less prone to seek, by their own initiative, the health care system for depressive symptoms. Such symptoms often have their onset later than symptoms of neurodevelopmental disorders, which debut in childhood and which might be brought to the attention of the health care system by family members. If this finding is replicated, more focus on detecting depressive symptoms in males with CL would be indicated. The increased risk of depression among males with $\mathrm{CPO}$ could be explained by secondary factors due to an underlying neuropsychiatric disorder. There is evidence that depression is the most common comorbidity among individuals with neuropsychiatric disorders, especially when transitioning from adolescence to adulthood. ${ }^{39}$ A combination of being less prone to seek care for treatment and an undetected neuropsychiatric disorder leading to high levels of distress could possibly be an explanation to the higher rates of suicide among OFC in Denmark; if so, that would indicate the importance of consistent follow-up and screening of children with OFC for neurodevelopmental disorders.

Finally, our sex-specific analyses revealed significant interactions with higher risks among females with OFC for any psychiatric disorder, intellectual disability, language disorders, ASD, and other behavioral disorders. A similar pattern was seen among children with CL and CLP, 
although it did not reach statistical significance. A possible explanation of a higher vulnerability of the female brain to the underlying neurodevelopmental mechanisms in OFC cannot totally be dismissed, but is not supported by the fact that no such patterns could be seen among children with $\mathrm{CPO}$, who had the highest risk increases for neurodevelopmental disorders. An alternative explanation might be a sex-specific diagnostic bias, with these disorders in females being less often diagnosed due to different presentation of symptoms or symptom severity. Recent studies have shown that females are more likely to be diagnosed with a neurodevelopmental disorder when they have additional comorbid features. ${ }^{40,41}$

The major strength of this study is the study design of a nationwide register-based cohort with a long follow-up time. This has provided us with a unique opportunity to study a large sample of patients with clefts, which is crucial when investigating relatively rare conditions. We were also able to explore possible different patterns among patients with different types of OFC malformations. Information collected from registers eliminates the risk of recall bias and selection bias. Another important strength is the availability of data used for our multivariate analyses to adjust for confounders. Moreover, the sibling cohort enabled us to match on many unmeasured factors, including cultural background, parental characteristics and child-rearing practices, and genetics. ${ }^{42}$

The diagnoses for identifying our study population and the psychiatric diagnoses were obtained from the National Patient Register. The validity of the psychiatric diagnoses in the NPR is considered high, as measured by moderate-tohigh positive predictive values for psychiatric diagnoses. ${ }^{43-45}$ It should be kept in mind, however, that psychiatric symptoms, such as mild depressive and anxiety symptoms, that are not severe enough to require psychiatric care are treated within the primary health care system, and those diagnoses registered in primary care are not included in the Swedish Patient Register.

The study also has some limitations. A number of individuals with OFC received several different ICD codes for their cleft type. This clinical misclassification may have resulted in results being diluted or exaggerated when stratified by cleft type.

There is a possibility that some individuals with OFC are classified as nonsyndromic, although they might have unidentified associated malformations or anomalies that could be associated with intellectual disability or other psychiatric disorders. Clinicians sometimes have difficulties identifying the correct code for genetic conditions and associated anomalies. This is expected to have occurred more often in the earlier-born individuals of the cohort, and we have adjusted for calendar year in our multivariate analyses to eliminate this risk. To decrease the risk even more for undetected syndromic OFC, we also decided to be overly inclusive in our definition of what diagnoses could indicate syndromic OFC, and we therefore adjusted for all congenital malformations and chromosomal abnormalities in our multivariate analysis.

Finally, there have been changes over time in the diagnostic criteria in the ICD-8, -9 , and -10 for specific neuropsychiatric diagnoses, which could possibly result in some uncertainty in the validity of our outcome measures. We decided to analyze the diagnosis mental retardation according to the ICD-8 together with the diagnosis intellectual disability according to ICD-9 and -10 . Because of the lack of specific ICD-8 codes for ADHD and ASD, the individuals who died before the ninth version of the ICD were therefore not included in the analyses for ADHD and ASD.

In conclusion, this large nationwide register study showed that children with nonsyndromic OFC in Sweden have an increased risk of being diagnosed with a neurodevelopmental disorder compared to children without clefts. The highest risk was for CPO, followed by CLP, whereas children with CL had the lowest, but still significant, risk increase. There was no overall risk increase for mood disorders or anxiety-related disorders, indicating that neurodevelopmental and neurological structural differences may play a major role for the observed associations. The full sibling analyses suggest that the higher risk of psychiatric disorders among individuals with orofacial clefts is unlikely to be explained by familial influences such as inherited genetic or shared environmental factors.

\footnotetext{
Accepted June 22, 2018.

Drs. Tillman, Hakelius, Ramklint, Ekselius, Nowinski, and Papadopoulos are with Uppsala University, Sweden. Mr. Höijer is with the Institute of Environmental Medicine, Karolinska Institutet, Solna, Sweden.

This study was financially supported by Medical Training and Research Agreement Funds (ALF) from Uppsala University Hospital. The funder had no role in study design, data collection and analysis, decision to publish, or preparation of the manuscript.

This study was presented as a poster at the American Academy of Child and Adolescent Psychiatry's 65th Annual Meeting, Seattle, WA, October 22-27, 2018, and as an abstract at the European Psychiatric Association's Annual Meeting, Nice, France, March 3-6, 2018.

Mr. Höijer served as the statistical expert for this research.

The authors wish to thank Clinical Genetician Eva-Lena Stattin, MD, PhD, of the Uppsala University Hospital, for assistance and genetic advice for this study.

Disclosure: Drs. Tillman, Hakelius, Ramklint, Ekselius, Nowinski, Papadopoulos and Mr. Höijer report no biomedical financial interests or potential conflicts of interest.

Correspondence to Karin Tillman, MD, Uppsala University, Child and Adolescent Psychiatry, Department of Neuroscience, Uppsala University Hospital entrance 10, Uppsala, 75185, Sweden; e-mail: karin.tillman@neuro.uu.se

0890-8567/\$36.00/๑2018 American Academy of Child and Adolescent Psychiatry. Published by Elsevier Inc. This is an open access article under the CC BY-NC-ND license (http://creativecommons.org/licenses/by-nc-nd/4.0/).

https://doi.org/10.1016/j.jaac.2018.06.024
} 


\section{REFERENCES}

1. Swanenburg de Veye HF, Beemer FA, Mellenbergh GJ, Wolters WH, Heineman-de Boer JA. An investigation of the relationship between associated congenital malformations and the mental and psychomotor development of children with clefts. Cleft Palate Craniofac J. 2003; 40:297-303.

2. Collander Farzaneh F. Cleft lip and palate: clinical studies regarding speech and facial growth. Lund, Sweden: Malmö University Hospital, Lund University; 2009.

3. Beriaghi S, Myers SL, Jensen SA, Kaimal S, Chan CM, Schaefer GB. Cleft lip and palate: association with other congenital malformations. J Clin Pediatr Dent. 2009;33:207-210.

4. Kallen B, Harris J, Robert E. The epidemiology of orofacial clefts. 2. Associated malformations. J Craniofac Genet Dev Biol. 1996;16:242-248.

5. Goodacre TS, Marc C. Cleft lip and palate: current management. Paediatrics Child Health. 2008;18:283-292.

6. Burdi AR. Cleft Lip and Palate. New York: Springer; 2006.

7. Chung KC, Kowalski CP, Kim HM, Buchman SR. Maternal cigarette smoking during pregnancy and the risk of having a child with cleft lip/palate. Plast Reconstr Surg. 2000; 105:485-491.

8. Little J, Cardy A, Arslan MT, Gilmour M, Mossey PA. Smoking and orofacial clefts: a United Kingdom-based case-control study. Cleft Palate Craniofac J. 2004;41:381-386.

9. Yazdy MM, Honein MA, Rasmussen SA, Frias JL. Priorities for future public health research in orofacial clefts. Cleft Palate Craniofac J. 2007;44351-44357.

10. Eliasson MJ. Neuropsychological perspectives of cleft lip and palate. In: Bardach J, Hughlett L, Morris, eds. Multidisciplinary Management of Cleft Lip and Palate. Philadelphia: W. B. Saunders; 1990.

11. Richman LC, McCoy TE, Conrad AL, Nopoulos PC. Neuropsychological, behavioral, and academic sequelae of cleft: early developmental, school age, and adolescent/young adult outcomes. Cleft Palate Craniofac J. 2012;49:387-396.

12. Richman LC, Ryan SM. Do the reading disabilities of children with cleft fit into current models of developmental dyslexia? Cleft Palate Craniofac J. 2003;40:154-157.

13. Broder HL, Richman LC, Matheson PB. Learning disability, school achievement, and grade retention among children with cleft: a two-center study. Cleft Palate Craniofac J. 1998;35:127-131.

14. Richman LC, Eliason MJ, Lindgren SD. Reading disability in children with clefts. Cleft Palate J. 1988;25:21-25.

15. Richman LN, Nopoulos N. Neuropsychological and neuroimaging aspects of clefting. In: Kirschner JELRE, ed. Comprehensive Cleft Care. New York: Springer; 2008.

16. Christensen K, Mortensen PB. Facial clefting and psychiatric diseases: a follow-up of the Danish 1936-1987 Facial Cleft cohort. Cleft Palate Craniofac J. 2002;39: 392-396.

17. Christensen K, Juel K, Herskind AM, Murray JC. Long term follow up study of survival associated with cleft lip and palate at birth. BMJ. 2004;328:1405.

18. Pedersen DA, Wehby GL, Murray JC, Christensen K. Psychiatric diagnoses in individuals with non-syndromic oral clefts: a Danish population-based cohort study. PLoS One. 2016;11:e0156261.

19. Nilsson S, Merlo J, Lyberg-Ahlander V, Psouni E. Psychotropic drug use in adolescents born with an orofacial cleft: a population-based study. BMJ Open. 2015;5:e005306.

20. Axelsson O. The Swedish Medical Birth Register. Acta Obstet Gynecol Scand. 2003;82: 491-492.

21. Socialstyrelsen. The Swedish National Patient Register. 2017. Available at: http://www. socialstyrelsen.se/register/halsodataregister/patientregistret/inenglish. Accessed May 30, 2018.

22. Socialstyrelsen. The Swedish Cause of Death Register. 2016. Available at: http://www. socialstyrelsen.se/register/dodsorsaksregistret. Accessed May 30, 2018.

23. Ekbom A. The Swedish Multi-generation Register. Methods Mol Biol. 2011;675: 215-220.
24. StataCorp. Stata Statistical Software: Release 15 [computer program]. College Station, TX: StataCorp; 2017

25. Williams RL. A note on robust variance estimation for cluster-correlated data. Biometrics. 2000;56:645-646.

26. Cross-Disorder Group of the Psychiatric Genomics Consortium. Identification of risk loci with shared effects on five major psychiatric disorders: a genome-wide analysis. Lancet. 2013;381:1371-1379.

27. Forero DA, Herteleer L, De Zutter S, et al. A network of synaptic genes associated with schizophrenia and bipolar disorder. Schizophr Res. 2016;172:68-74.

28. Kapp-Simon KA, Simon DJ, Kristovich S. Self-perception, social skills, adjustment, and inhibition in young adolescents with craniofacial anomalies. Cleft Palate Craniofac J. 1992;29:352-356.

29. Millard T, Richman LC. Different cleft conditions, facial appearance, and speech: relationship to psychological variables. Cleft Palate Craniofac J. 2001;38:68-75.

30. Richman LC. Self-reported social, speech, and facial concerns and personality adjustment of adolescents with cleft lip and palate. Cleft Palate J. 1983;20:108-112.

31. Nopoulos P, Langbehn DR, Canady J, Magnotta V, Richman L. Abnormal brain structure in children with isolated clefts of the lip or palate. Arch Pediatr Adolesc Med. 2007;161:753-758.

32. Adamson CL, Anderson VA, Nopoulos P, Seal ML, Da Costa AC. Regional brain morphometric characteristics of nonsyndromic cleft lip and palate. Dev Neurosci. 2014; 36:490-498.

33. Chollet MB, DeLeon VB, Conrad AL, Nopoulos P. Morphometric analysis of brain shape in children with nonsyndromic cleft lip and/or palate. J Child Neurol. 2014;29: 1616-1625.

34. Nopoulos P, Swayze V, Flaum M, Ehrhardt JC, Yuh WT, Andreasen NC. Cavum septi pellucidi in normals and patients with schizophrenia as detected by magnetic resonance imaging. Biol Psychiatry. 1997;41:1102-1108.

35. Bodensteiner JB, Schaefer GB, Craft JM. Cavum septi pellucidi and cavum vergae in normal and developmentally delayed populations. J Child Neurol. 1998;13:120-121.

36. Mueller AA, Sader R, Honigmann K, Zeilhofer HF, Schwenzer-Zimmerer K. Central nervous malformations in presence of clefts reflect developmental interplay. Int J Oral Maxillofac Surg. 2007;36:289-295.

37. Kjaer I. Human prenatal craniofacial development related to brain development under normal and pathologic conditions. Acta Odontol Scand. 1995;53:135-143.

38. Marcucio RS, Cordero DR, Hu D, Helms JA. Molecular interactions coordinating the development of the forebrain and face. Dev Biol. 2005;284:48-61.

39. Lugnegard T, Hallerback MU, Gillberg C. Psychiatric comorbidity in young adults with a clinical diagnosis of Asperger syndrome. Res Dev Disabil. 2011;32:1910-1917.

40. Lai MC, Lombardo MV, Auyeung B, Chakrabarti B, Baron-Cohen S. Sex/gender differences and autism: setting the scene for future research. J Am Acad Child Adolesc Psychiatry. 2015;54:11-24.

41. Polyak A, Rosenfeld JA, Girirajan S. An assessment of sex bias in neurodevelopmental disorders. Genome Med. 2015;7:94.

42. Frisell T, Oberg S, Kuja-Halkola R, Sjolander A. Sibling comparison designs: bias from non-shared confounders and measurement error. Epidemiology. 2012;23:713-720.

43. Ludvigsson JF, Andersson E, Ekbom A, et al. External review and validation of the Swedish National Inpatient Register. BMC Public Health. 2011;11:450.

44. Sellgren C, Landen M, Lichtenstein P, Hultman CM, Langstrom N. Validity of bipolar disorder hospital discharge diagnoses: file review and multiple register linkage in Sweden. Acta Psychiatr Scand. 2011;124:447-453.

45. Ruck C, Larsson KJ, Lind K, et al. Validity and reliability of chronic tic disorder and obsessive-compulsive disorder diagnoses in the Swedish National Patient Register. BMJ Open. 2015;5:e007520. 